\title{
Knowledge Based System for Business Software
}

\author{
Dr. Velayutham Pavanasam
}

Associate Professor, Department of Computer Science and Engineering

St. Joseph College of Engineering and Technology, Tanzania, Africa

\begin{abstract}
The objective of this work is to propose a knowledge based system for an effective design and fault free development process of any business software. In this proposed work, the software requirement specifications are treated with proportionate associativity in a particular context. All such requirement information is fed into a knowledge base system in multiple contexts that focuses upon the customer values with respect to development time, cost and maintenance parameters. Various customer, system and environment requirements are analyzed with different contexts and information available in the knowledge base is considered as a knowledge engine to develop a robust software design. Knowledge based system for changing requirements of business software is being evaluated to arrive at a safe and effective design.
\end{abstract}

Keywords: knowledge base, requirement engineering, software design, business software, requirement specifications

\section{Introduction}

Knowledge base is a database used to share knowledge. It provides a mean for information to be collected, shared and utilised. Thus knowledge base is used to collect, organize and retrieve knowledge. Knowledge level process model of requirement engineering is a set of abstractions that represents requirement elicitation, manipulation and maintenance processes. Thus knowledge-level process is important for its decomposition based on separation of concerns and realization of applicable characterization [1]. Difficulties associated with the engineering of complex systems have manifested themselves in a crisis of software productivity. Knowledge-based software engineering attempts these ever-increasing difficulties in developing, extending and maintaining software [2]. In Kreutzer model, the life cycle model of a simulation project begins with system identification and concludes with output analysis commonly referred to as the nine aspects of simulation methodology. There is a transition from programming language specification to conceptual model specification. The problem is that there isn't any explicit referenc e for model requirements, requirement specification or any other related terms [3]. In Balci-Nance model, the various activities are identified starting with problem recognition till the use of results in the decision support. Problem formulation, model development and integrated decision support are the three phases in this model. The disadvantage of Balci-Nance model is that there isn't any direction specified to develop, analyze or use these requirements [4]. Sargent model is an effective life-cycle model of a simulation model of a continually changing complex system. In this model, theory, conceptual model and operational results are the three forms of validation technique. The drawback of this model is that the description of activities doesn't go in -depth to specify the model requirements [5]. Goal-Oriented Requirement Engineering (GORE) is concerned with the use of goals for eliciting, elaborating, specifying, analyzing, negotiating, documenting and modifying requirements. It is used to identify the goals and develop a model. In a GORE process, functional goals are used to build operational models like use cases and state machine models whereas quality goals models are used to compare alternative options, select preferred ones and impose further constraints on goal operationalizations [6]. Aspects-Oriented Requirement Engineering (AORE) refers to set of activities that are concerned with identifying, specifying and representing crosscutting concerns and their modularization into separate functional units. It provides improved support for separation of crosscutting functional and non-functional properties during requirement engineering and understanding of the problem and the ability to reason about it. There is explicit separation of the composition specifications where these compositions are always intertwined with the goal graphs in goal-oriented techniques [7]. A formal description method of requirement engineering process Requirements Engineering Process Metamodel (REPM) has been proposed. The formal definition of REPM is provided and three typical requirements are specified. These three requirement engineering processes namely top-down, middle-out and bottom-up processes are defined and specifically analysed. REPM is a simple method available for describing the different types of processes and a new integrated requirement engineering process is defined using REPM [8]. Knowledge Acquisition and Sharing for Requirement Engineering (KARE) is another important approach for the requirement specification of one-of-a-kind complex systems. KARE provides an interface for system requirement review and has two main integrated components which are requirement engineering process and knowledge engineering process. KARE approach consists of important tasks such as requirement elicitation, requirement analysis and requirement negotiation process. A new customisable model comprising the various tasks, interferences as well as the domain level knowledge categories specific to the system engineering activity has been successfully implemented [9]. A knowledge based requirement engineering framework for emergency management system has been proposed as an integrated model of expert system and meta-modeling of target services to arrive at a reusable design and deployment of a flood situation service in emergency management system software. The domain expert, meta-modeler and decision support modules are incorporated together as knowledge 


\section{International Journal of Science and Research (IJSR) \\ ISSN (Online): 2319-7064 \\ Index Copernicus Value (2013): 6.14 | Impact Factor (2014): 5.611}

based framework to arrive at a safe and effective design for an emergency management system [10].

\section{Knowledge Base for Requirement Engineering}

It is necessary to know the difference between data and information commonly occurring in any software application. Data is raw information that is collection of facts which must be processed to obtain information. Information is derived by associating facts within a given context. Knowledge base represents facts about the real world that is the information obtained in one context with the information obtained in different context. Knowledge is two-dimensional. The information collected based upon a variety of related and unrelated topics are connected to form a body of facts termed knowledge. The various pieces of information can be combined to formulate a representation of knowledge. Using this knowledge, a business opportunity may emerge. New learning modes can be developed which are efficient and cost effective than other approaches. Knowledge-based system focuses on the systems that use knowledge-based methods to support action, learning as well as decision-making. Context and data can be represented as shown in Figure 1.

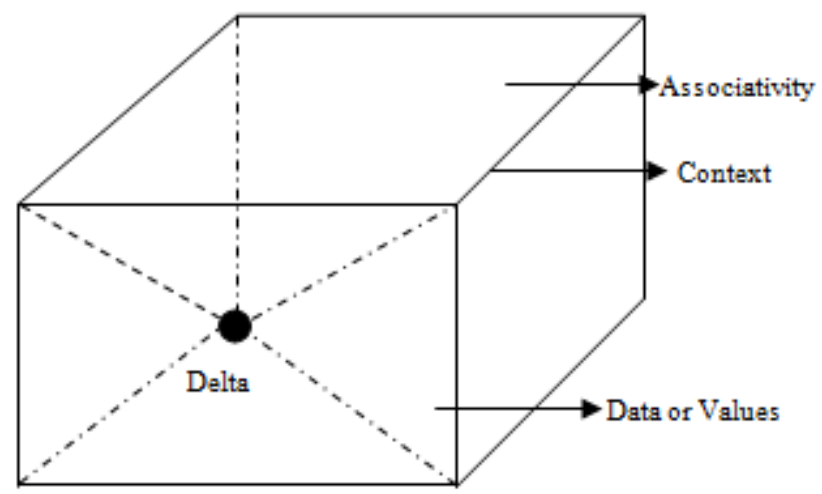

Figure 1: Context Data Representation

If customer requirements (CR), system requirements (SR), and environment requirements (ER) are passed as input parameters through knowledge based system then the final output will be a 16 bit software design as shown in Figure 2.

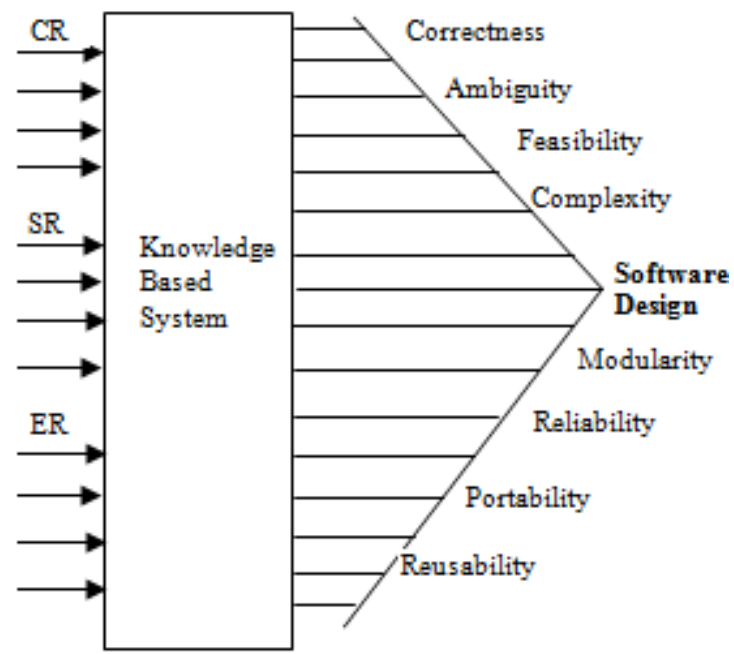

Figure 2: Knowledge Based System
Knowledge Based System (KBS) consists of various factors such as:

- Aspect: KBS must be focused on a specific task

- Context: Key features of the wider organization to put the problems and opportunities into perspective

- Resources: Resources are utilized for business processes such as information systems and technology

- Strategy: Important policies must be included in business processes

- Rules: Rules depict and enforce the policies of an organization

- Constraints: Stands in the way of customers using them

\section{Business Software Requirements}

Consider various software packages such as Enterprise Resource Planning (ERP), System Analysis and Program Development (SAP), Supply Chain Management (SCM) and Customer Relation Management (CRM) for business software requirements. The various parameters can be rated correspondingly in the scale of 1-10 where 1 is the lowest priority and 10 is the highest priority with respect to the packages as shown in Table 1.

\section{Customer Requirements}

- Functionality (FN): Component must contain the required functionality

- Security (SE): Security must be provided for data

- Performance (PE): High end performance must be provided

- Interoperability (IN): Important policies must be included in business processes

- Scalability (SC): Characteristic of a system or model that describes its capability to perform under an increased workload

\section{System Requirements}

- Browsing Speed (BS): High end systems are necessary for fast manipulation of data

- Plug-Ins (PI): A number of plug-ins must be available for large users

- Reliability (RE): System must be reliable to work with under any conditions

- Modularity (MO): System must be divided into several modules

\section{Environment Requirements}

- Data Transfer Rate (DT): Data must be transferred at a faster rate

- Bandwidth (BW): High bandwidth is essential for information retrieval

- Channel Type (CT): Various types of channels must be specified 


\section{International Journal of Science and Research (IJSR) \\ ISSN (Online): 2319-7064}

Index Copernicus Value (2013): 6.14 | Impact Factor (2014): 5.611

Table 1: Requirement Software Packages

\begin{tabular}{|c|c|c|c|c|c|c|c|c|c|c|c|c|}
\hline Requirements & \multicolumn{4}{|c|}{ Customer Requirements } & \multicolumn{4}{c|}{ System Requirements } & \multicolumn{4}{c|}{ Environment Requirements } \\
\hline Domain/ Packages & $F N$ & $S E$ & $P E$ & $I N$ & $S C$ & $B S$ & $P I$ & $R E$ & $M O$ & $D T$ & $B W$ & $C T$ \\
\hline ERP & 8 & 8 & 7 & 5 & 4 & 7 & 7 & 9 & 10 & 7 & 5 & 5 \\
\hline SAP & 8 & 7 & 8 & 6 & 5 & 6 & 8 & 8 & 10 & 7 & 6 & 4 \\
\hline SCM & 9 & 8 & 7 & 6 & 4 & 6 & 8 & 9 & 10 & 7 & 6 & 5 \\
\hline CRM & 8 & 8 & 7 & 6 & 5 & 7 & 8 & 9 & 10 & 6 & 5 & 5 \\
\hline
\end{tabular}

\section{Conclusion}

Knowledge based system is a software system with an explicit, declarative description of knowledge for a certain application. Knowledge based system for any business software has been successfully proposed. This is necessary to arrive at a safe as well as effective design. When customer, system and environment requirements are passed as input parameters through knowledge based system, 16 bit software design is obtained as output. Maintenance, cost and time parameters have been considered wherein the requirements are fed into a knowledge base system. Various factors like aspect, context, strategy, rules, constraints and resources are considered in a knowledge based system. Software packages such as ERP, SAP, SCM and CRM for business software requirements are tabulated against customer, system and environment requirements for parameters such as functionality, performance, browsing speed, reliability etc. The various parameters have been rated in the scale of 1-10. The tabulated results shows the values obtained for software packages against the corresponding parameters included under customer, system and environment requirements. Thus knowledge based system for business software with changing requirements has been successfully evaluated to arrive at a safe, effective and robust software design.

\section{References}

[1] D.E. Herlea, C.M. Jonker et. al. "A Computational Knowledge Level Model of Requirement Engineering," The International Journal of Software Engineering and Knowledge Engineering, vol 12, pp. 41-75, 2002.

[2] Website http://acs/.cs.uiuc.edu/kbse

[3] W. Kreutzer, "System Simulation - Programming Styles and Languages," New York : Addison Wesley, 1986.

[4] O. Balci, R.E. Nance, "Simulation Model Development Environments: A Research Prototype," Journal of the Operational Research Society (JORS), vol. 38, no 8, pp. 753-763, 1987.

[5] R.G. Sargent, "Some Approaches and Paradigms for Verifying and Validating Simulation Models," IEEE Winter Simulation Conference, Arlington, VA, pp.106-114, 2001.

[6] A. Van Lamsweerde, "Goal Oriented Requirements Engineering : A Roundtrip from Research to Practice," $12^{\text {th }}$ IEEE International Requirements Engineering Conference, pp. 249-262, 2004.

[7] Awais Rashid, "Aspect-Oriented Requirements Engineering: An Introduction," $16^{\text {th }}$ IEEE International Requirements Engineering Conference, pp. 306-309, 2008.

[8] Xuping Jiang, "Modelling and Application of Requirements Engineering Process Metamodel," IEEE International Conference pp. 998-1001, 2008.
[9] S. Ratchev, E. Urwin, et. al., "Knowledge Based Requirement Engineering for one-of-a-kind Complex Systems," International Journal of Knowledge-Based Systems, Elsevier, vol. 12, no 1, pp. 1-5, 2003.

[10] V. Pavanasam, C. Subramaniam et. al. "Knowledge Based Requirement Engineering Framework for Emergency Management System", $2^{\text {nd }}$ IEEE International Conference on Computer Engineering and Applications (ICCEA), pp. 601-605, 2010.

\section{Author Profile}

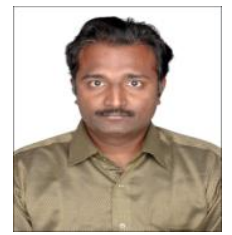

Dr. Velayutham Pavanasam received Ph.D. Degree in Computer Science from Satyabhama University, Chennai, India. He is currently working as Associate Professor in Department of Computer Science and Engineering at St. Joseph College of Engineering and Technology, Tanzania, Africa. He has 18 years of teaching experience and guided many academic projects both for graduate and postgraduate students. He has presented his research papers abroad in various countries like Indonesia and Thailand. His research area of interest includes Software Engineering, Software Reliability and Software Quality Assurance and Testing 